\title{
Fast simulation and optimization tool to explore selective neural stimulation
}

\author{
Mélissa Dali (1), Olivier Rossel (1), David Guiraud (1)
}

(1) INRIA-LIRMM - Université de Montpellier, Campus Saint Priest-Montpellier, France

This article is distributed under the terms of the Creative Commons Attribution Noncommercial License (CC BY-NC 4.0) which permits any noncommercial use, distribution, and reproduction in any medium, provided the original author(s) and source are credited.

\begin{abstract}
In functional electrical stimulation, selective stimulation of axons is desirable to activate a specific target, in particular muscular function. This implies to simulate a fascicule without activating neighboring ones i.e. to be spatially selective. Spatial selectivity is achieved by the use of multicontact cuff electrodes over which the stimulation current is distributed. Because of the large number of parameters involved, numerical simulations provide a way to find and optimize electrode configuration. The present work offers a computation effective scheme and associated tool chain capable of simulating electrode-nerve interface and find the best spread of current to achieve spatial selectivity.
\end{abstract}

Key Words: spatial selectivity, cuff electrode, quasi-static approximation

Implanted electrical stimulation is an approach used for many years with success to restore motor or organic function. The main objective of FES [e.g. Functional Electrical Stimulation (FES)] is to provide efficient recruitment of nervous fiber as the natural behavior would achieve. In distal regions ${ }^{1-3}$ it exists a spatial organization of fascicles that target specific pools of motor neurons. To independently activate muscles, a control of activation by spatial selectivity of axons is required. Selectivity as defined in ${ }^{4}$ is "the ability to activate one population of neurons without activating neighboring ones". A way to focus activation profile is the use of multicontacts cuff electrode with multipolar configuration of currents. ${ }^{5-7}$. Number of contacts, their size and the current distribution have a large influence on axons regional activation. Our technology 8 is able to provide multipolar current configuration and complex waveforms profile. Since the number of parameters is very huge, testing all the current profiles in experiments is not realistic, in particular for clinical applications. Numerical simulations can help to find optimal configurations by modeling interface between electrode and nerve. A 2-part process is commonly used to simulate electrical stimulation. First, computation of the electric field generated by the electrode within the nerve, assuming that nerve is a volume conductor. Second, determination of axons excitation within the nerve based on the results of the previous computations. These numerical investigations are quite long, and need for experts to be carried out. All-in-one fast tool chain was created to overcome these issues. This software is divided in two parts: Computation of electric field performed by OpenMEEG ${ }^{9}$ and activation / optimization of current spreading computed through Matlab scripts.

\section{Materials and Methods}

The software MOS2SENS (from Model Optimization and Simulation To Selective Electrical Neural Stimulation) is an adjustment support tool for neuroprosthetic devices. It models and optimizes the current injected by cuff electrodes inside the nerve in order to activate selective fiber targets in terms of spatial criterion. There are two software that perform the following functions:

- Generation of 3D geometric model of nerve and cuff electrode

- Mathematical description of the link between stimulation currents and extracellular voltage inside the nerve

- Nerve fiber activation prediction based on the current stimulation

- Optimization of the current injected according to the chosen target and cost function

The electrical potential i.e. extracellular voltage is computed with OpenMEEG open source software, using the BEM [e.g. Boundary Element Method (BEM)] to solve Laplace equation, under quasi-static approximation. $^{10,11}$ Determination of activation was based on activating function. ${ }^{12}$ Optimization is 


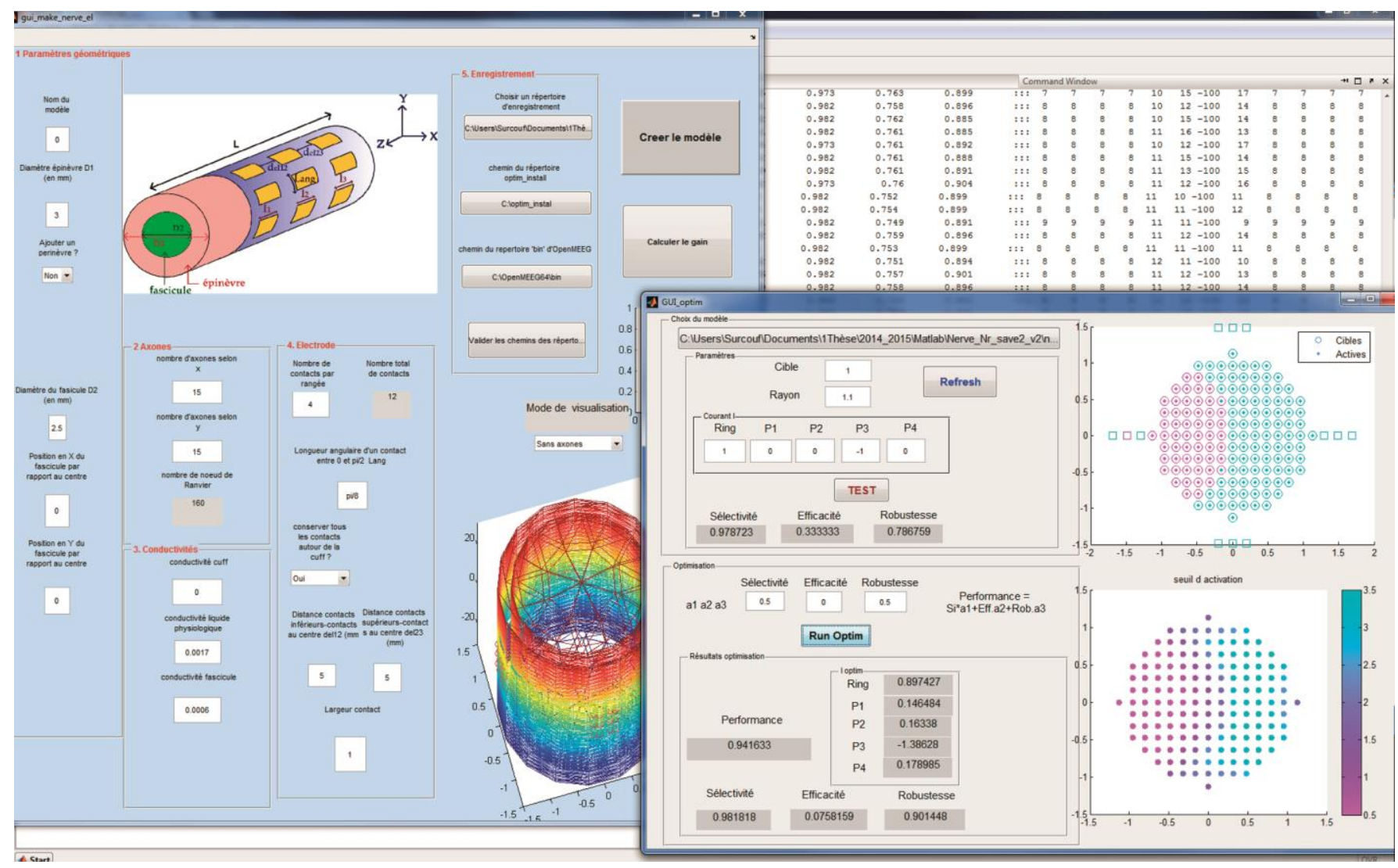

Fig 1. Graphical user interface

performed in term of selectivity, efficiency and robustness. The software was implemented in Matlab with graphical user interface (Figure 1).

\section{Results}

\section{A. 3D Model settings}

User can set the following geometrical parameters to simulate 3D model of the nerve: number of layers (minimum an epineurium and a fascicle), diameter of each layer, number of nervous fibers inside a fascicle (Nbfib). It's possible to set conductivity for each layer, but only isotropic. The 3D nerve model is made up of nested cylinders which surfaces are represented by bounded triangular meshes. The inner cylinder represents a fascicle that contains axons. The outer cylinder represents the connective tissue that surrounds the fascicle. It's possible to simulate perineurium between fascicle and epineurium. The conductivities of the media are supposed piece-wise constant. Default values are taken from litterature ${ }^{13,14}$ and user have the possibility to modify them. Only myelinated fibers are considered, therefore, we only need to know value of potential field at the Ranvier Nodes. The Ranvier nodes are represented as a grid of point in space (Nbfib, 160 nodes per fiber).
With regards to the electrode, user can choose number, size and position of contacts around the nerve. By default, number of contact is set to 12 but it's possible to increase or decrease this number, and simulate the case where the electrode's span does not fit exactly to the nerve diameter. Once parameters are set, OpenMEEG computes a lead field matrix describing the mathematical link between electrode and node of Ranvier.

\section{B. Activation and optimization settings}

Once computation of lead field matrix is achieved, user can visualize fibers activation related to the distribution of currents. Activation is shown across a $2 \mathrm{D}$ cross section of nerve by taking the maximum of activating function along each fiber. First, user chooses a target area within the nerve. He can manually test different spreads of the currents, i.e., multipolar configurations, and visualize performances in terms of spatial selectivity, efficiency and robustness indexes. Then, user can choose weights for indexes and find the optimized solution linked to the cost function. The results are expressed in terms of current distribution and total injected charges. 


\section{Discussion}

We created software to evaluate quickly the effects of multipolar configurations on spatial selectivity and optimize spread of currents through the contacts of a CUFF electrode. Computation of electric field within the nerve represented by two layer (endoneurium and epineurium) is achieved in 25 min for 333 fibers with 160 node of Ranvier, this is quite short compared to other method for instance FEM. ${ }^{15}$ Computation of activation takes less than $1 \mathrm{~s}$ by using predictive model of activating function. ${ }^{12}$ Optimization of current spreading is obtained instantaneously (less than 1s). Computation of potential was made under quasi-static approximation: propagation effects and inductive effects are negligible, capacitive effects are reasonably ignored. ${ }^{10,11}$

However, the last assumption is correct if capacitive current of the media is negligible compared to resistive current, which are frequency dependent. User must consider this criterion before to use the program. The model was built to study current-controlled stimulation electrode. In this case, electrode capacitance could be ignored and electrode-tissue interface was not represented.

In conclusion, MOS2SENS is a powerful tool to scan optimal spatial configurations of 12-pole electrode. MOS2SENS is filed in the APP [e.g. Agency for the Protection of Programs (APP)] under the identifier: IDDN.FR.001.490036.000.S.P.2014.000.31230. Future work will provide the possibility to model anisotropy, and more complex geometry such as multifascicles. Results of simulations will be shown and a demo would be provided. Finally, experimental work is under design, to validate the results of simulations.

\section{Contributions}

MD Software development, publication writing; OR Software development, publication writing; DG Supervision of software development, publication drafting, publication review.

\section{Acknowledgements}

This work was supported by BPI France, Grant "Intense" Number \#6354. Thanks to Maureen Clerc for her help on the use of OpenMEEG. Paper prepared for the 2016IFESS Conference.

\section{Conflict of Interest}

The authors declare no potential conflict of interests.

\section{Corresponding Author}

Mélissa Dali, INRIA-LIRMM - Université de Montpellier, Campus Saint Priest - Bat 5 - Bureau 03/232, 860 Rue de St Priest, 34095 Montpellier cedex 5

- France.

E-mail: melissa.dali@inria.fr

E-mails of coAuthors

David Guiraud: david.guiraud@inria.fr Olivier Rossel: olivier.rossel@lirmm.fr

\section{References}

1. Sunderland S, Walshe F. Nerves and Nerve Injuries. E. \& S. Livingstone, Edinburgh and London; 1968.

2. Jabaley ME, Wallace WH, Heckler FR. Internal topography of major nerves of the forearm and hand: a current view. J Hand Surg Am 1980;5:118. doi:10.1097/00006534-198011000-00072.

3. Gustafson KJ, Pinault GCJ, Neville JJ, et al. Fascicular anatomy of human femoral nerve: Implications for neural prostheses using nerve cuff electrodes. J Rehabil Res Dev 2009;46:973. doi:10.1682/JRRD.2008.08.0097.

4. Grill WM, Mortimer JT. Stimulus waveforms for selective neural stimulation. IEEE Eng Med Biol Mag 1995;14:375-385. doi:10.1109/51.395310.

5. Deurloo KEI, Holsheimer J, Boom HBK. Transverse tripolar stimulation of peripheral nerve: a modelling study of spatial selectivity. Med Biol Eng Comput 1998;36:66-74. doi:10.1007/BF 02522860.

6. Sweeney JD, Ksienski DA, Mortimer JT. A nerve cuff technique for selective excitation of peripheral nerve trunk regions. IEEE Trans Biomed Eng 1990;37:706-15. doi:10.1109/10.55681.

7. Ordelman SCMA, Kornet L, Cornelussen R, et al. Selectivity for Specific Cardiovascular Effects of Vagal Nerve Stimulation With a Multi-Contact Electrode Cuff. IEEE Trans Neural Syst Rehabil Eng 2013;21:32-6. doi:10.1109/TNSRE.2012. 2214058.

8. Andreu D, Guiraud D, Souquet G. A distributed architecture for activating the peripheral nervous system. J Neural Eng 2009;6:026001. doi:10.1088/ 1741-2560/6/2/026001.

9. Gramfort A, Papadopoulo T, Olivi E, Clerc M. OpenMEEG: opensource software for quasistatic bioelectromagnetics. Biomed Eng Online 2010;9:45. doi:10.1186/1475-925X-9-45.

10. Plonsey R, Heppner DB. Considerations of quasistationarity in electrophysiological systems. Bull Math Biophys 1967;29:657-64. doi:10.1007/ BF02476917.

11. Bossetti CA, Birdno MJ, Grill WM. Analysis of the quasi-static approximation for calculating potentials generated by neural stimulation. J Neural Eng 2008;5(1):44-53. doi:10.1088/17412560/5/1/005.

12. Rattay F. Analysis of models for external stimulation of axons. IEEE Trans Biomed Eng 1986;33:974-7. doi:10.1109/TBME.1986.325670.

13. Choi AQ, Cavanaugh JK, Durand DM. Selectivity of multiple-contact nerve cuff electrodes: a simulation analysis. IEEE Trans. Biomed Eng 2001;48:165-72. doi:10.1109/10. 909637. 


\section{Tool chain to explore selective neural stimulation}

Eur J Transl Myol 26 (3) 215-218

14. Geddes LA, Baker LE. The specific resistance of biological material - A compendium of data for the biomedical engineer and physiologist. Med Biol Eng 1967;5:271-93. doi:10.1007/BF 02474537.

15. Jacquir S, Fruitet J, Guiraud D, Clerc $M$. Computation of the electrical potential inside the nerve induced by an electrical stimulus. In: Annual International Conference of the IEEE Engineering in Medicine and Biology Proceedings 2007:1711-4. doi:10.1109/IEMBS. 2007.4352639 . 